\title{
A Study on Dual-Polarization Broadband Antenna Array Covered with EM Wave Absorber
}

\author{
Wen-Yu Lee ${ }^{1, \mathrm{a}}$, Hsin-Piao Lin ${ }^{1, \mathrm{~b}}$, Ding-Bing Lin ${ }^{2, \mathrm{c}}$ \\ ${ }^{1}$ Department of Electronic Engineering, Taipei University of Technology, No.1, Section 3, Zhongxiao East Road, Taipei City, \\ Taiwan, R.O.C. \\ ${ }^{2}$ Department of Electronic Engineering, National Taiwan University of Science and Technology, No.43, Section 4, Keelung \\ Road, Daan District, Taipei City, Taiwan, R.O.C.

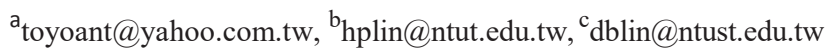

\begin{abstract}
The CTIA test plan clearly specifies that up to 16 sets of dual-polarized antennas must be installed in the MIMO OTA EM wave anechoic chamber as an important condition for multi-channel OTA measurement. In this paper the authors use two FRP-4 PCBs each with parallel Vivaldi antennas and assembly crossing at $90^{\circ}$ to form a dual-polarized compact broadband antenna with a very small cross-sectional area, allowing it to operate over a wide range from 0.7 to $6.0 \mathrm{GHz}$. They also use a microstrip power splitter to design the antenna array. The authors are very skillful in using two parallel antennas on each PCB to increase the directional gain of EM wave propagation, which also improves the isolation between vertical and horizontal polarized antennas. In this paper due to the authors' special design method, the isolation between the vertical and horizontal polarized antennas is as high as approximately $40 \mathrm{~dB}$. The authors also use a foam cone EM wave absorber to cover this compact wideband antenna array to prevent multipath reflections and reduce signal interference outside the propagation direction of the antenna array. When the MIMO OTA anechoic chamber is installed with multiple antennas designed by the authors and simultaneously transmits EM wave, stable measurement results can be obtained.
\end{abstract}

Keywords: Antenna array, Vivaldi, Dual polarization, $B$ roadband antenna

\section{Introduction}

In mobile communications, multiple-input multipleoutput over-the-air (MIMO OTA) measurements apply electromagnetic (EM) wave anechoic chambers, as shown in Fig. 1. In 2016, CTIA-The Wireless Association introduced regulations regarding EM wave anechoic chambers. At least 8-16 dual-polarized antennas must be installed in an EM wave anechoic chamber and multichannel communications measurements must be conducted using regulated communication models. Conventional EM wave anechoic chambers mainly use broadband open boundary horn antennas operating at a frequency range of $0.7-6.0 \mathrm{GHz}$ (Fig. 2). However, conventional open boundary horn antennas commonly used are made of aluminum. These types of antennas are bulky and possess a large cross-section area along the propagation direction, as shown in Fig. 2, thereby resulting in a multipath reflection interference of multiple sets of EM wave propagation. Such mutual interference has cross effects on the isolation between antennas and causes measurement errors (Fig. 3). In this study, an antenna with a smaller cross-section area along the propagation direction than that of a conventional open boundary horn antenna was devised (Fig. 4). Foaming EM wave absorbers were used to coat the antenna adequately (Fig. 5) to reduce the multipath reflections of signals and mutual interferences. The configuration of the proposed antenna is as follows: Two printed circuit boards (PCBs), which were designed with two parallel Vivaldi antennas on a plane to form an array [1] (as shown in Fig. 6), were assembled to form a cross at $90^{\circ}$, creating a dual-port antenna array with dual-polarization function in vertical and horizontal planes. A square metal reflector was installed at the bottom of the antenna array (as shown in Fig. 7). A well-designed microstrip power splitter was used as the coupling and feed points of vertical and horizontal signal sources.

In 1979, Gibson developed a broadband exponentially tapered microstrip slot antenna, which is called the Vivaldi antenna [2]. The open-end curvature of the Vivaldi antenna is exponentially tapered with a broad operating band of frequency, high directional gain, and horizontally or vertically symmetrical wave beams. The open boundary horn antenna used in the present study was fabricated using PCBs based on FR4 fiberglass substrates laminated with copper foils on both sides. The signal feeding microstrip lines and splitters were printed on the front side of the PCB, whereas the exponential Vivaldi open boundary antenna was printed on the other side. This configuration can function as the impedance transform grid when signals feed in splitters and the free space of radiation propagation. It also has the function of a signal coupling feed. The width of the slot at the narrow end of the antenna ${ }^{e e}$ s exponential open boundary can determine the cutoff frequency (maximum frequency), whereas the slot width at the broader end of the open boundary can determine the initial frequency (minimum frequency). Schaubert et al. arranged this type of antenna as array elements to form single- and dual-polarized arrays and used them in phased arrays [3]. These applications reveal that researchers have attained considerable achievements in the research of Vivaldi antennas. 


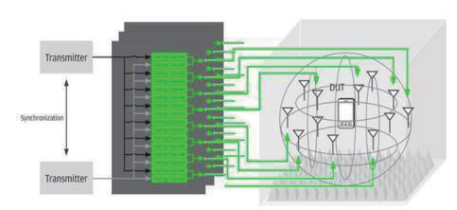

Fig. 1 MIMO OTA EM wave anechoic chamber installed with 16 sets of antennas.
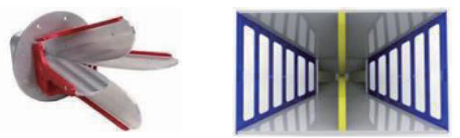

Fig. 2 Broadband open boundary horn antenna with a large cross-section area along the propagation direction.

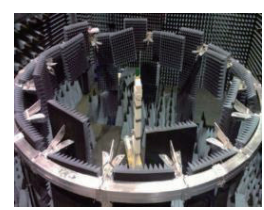

Fig. 3 MIMO OTA EM wave anechoic chamber installed with multiple sets of open boundary antennas.

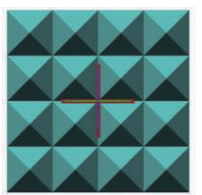

Fig. 4 Antenna with smaller cross-section area along the propagation direction proposed in this study.
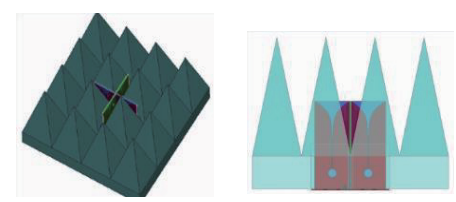

Fig. 5 Antenna encompassed by EM wave absorbers.

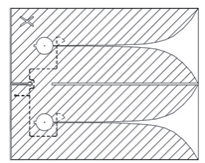

Fig. 6 Parallel Vivaldi antenna on a plane.

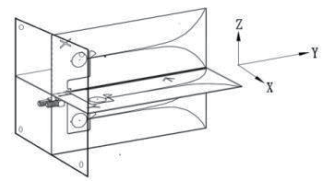

Fig. 7 Dual-port dual-polarized antenna array with a metal reflector.
Principle and Structure
A. Principle of operation
The Vivaldi antenna is a nonperiodic traveling wave antenna with tapering resonant frequencies. The exponentially tapered slot can radiate EM waves outward or receive them inward. To any operating point at the broadband operating band, resonance is formed only at regions where the exponential slot width approaches onefourth of the frequency wavelength; radiation is formed outside the slot space. Different from microstrip and whip antennas, the Vivaldi antenna presents radiative and resonant characteristics of a terminal radiation antenna, which enables surface waves to transmit to the open end and radiate. This type of antenna enhances directivity and gain. The most prominent advantage of the Vivaldi antenna lies in its wide operating broadband characteristics [4]. The Vivaldi antenna possesses excellent symmetrical radiation patterns on the main polarized horizontal and vertical planes (E-Plane and H-Plane, respectively) with its radiation polarization being linear. When PCBs with different dielectric constants are used to design a Vivaldi antenna, the PCB thickness and the size and shape of the exponentially tapered slot can change the half-power beam width and operating bandwidths. This study used a highly directive linear polarized antenna array that comprised two parallel Vivaldi antennas on the same plane [5], [6]. When the curvature of the exponentially tapered slot and the circular cavity size was adjusted as an impedance matching method to improve the Vivaldi antenna performance, the propagation characteristics and band width varied.

\section{B. Structure design analysis}

The structural design of the proposed antenna can be divided into four layers as follows: The first layer is the FR4 substrate plate. The second layer is the exponentially tapered slot antenna. The third layer is again the FR4 substrate. The fourth layer is a power splitter and copper-foil laminated microstrips for signal feeding [7], as shown in Fig. 8. The antenna body"s exponentially tapered slot structure was notched onto the copper foil lamination of the second layer. The curve of the tapered slot was composed of three parts: (1) a circular cavity for antenna impedance matching and adjustment, (2) a parallel slot as the signal feeding and coupling device that influences the insertion loss of the feeding signals, and (3) a circular sector primarily designed for propagation lines and antenna coupling, as illustrated in Fig. 9.

The operating bandwidth of the proposed antenna was determined using (1) the curvature of the exponentially tapered slot, (2) microstrip line design methods, (3) circular sector coupling design, (4) impedance matching of the circular cavity, and (5) thickness of the antenna body material and PCB dielectric constants. The method of incorporating impedance matching into the antenna design effectively expanded the antenna bandwidth and reduced reflection losses and improved impedance matching characteristics [8].

The curvature of the proposed exponentially tapered slot Vivaldi antenna affected the antennaes operating characteristics of broadband arrays. The curvature of critical 


\section{Recent Developments on Information and Communication Technology (ICT) Engineering- Meen, Yang \& Zhao ISBN: 978-981-14-2136-5}

design parameters was determined based on the following equations:

$$
\begin{aligned}
& y=c_{1} e^{R x}+c_{2} \\
& c_{1}=\frac{y_{2}-y_{1}}{e^{R x_{2}}-e^{R x_{1}}} \\
& c_{2}=\frac{y_{1} e^{R x_{2}}-y_{2} e^{R x_{1}}}{e^{R x_{2}}-e^{R x_{1}}}
\end{aligned}
$$

Because the medium between the slots was the FR4 substrate, the dielectric constant was 4.4. Therefore, the relationship between operating frequency $f H$ and slot width $W a$ is as follows:

$$
W a / 4=\frac{c}{f_{H} \sqrt{\varepsilon_{e}}}
$$

Fig. 10 shows that the antenna ${ }^{\text {ee }} \mathrm{s}$ exponentially tapered slot had two key parameters P1 $(\mathrm{x}, \mathrm{z})$ and P2 (x, z), respectively, as the start and end points of the curvature. $\mathrm{R}$ is the exponential change rate of the slot; Dr is the diameter of the circular cavity.

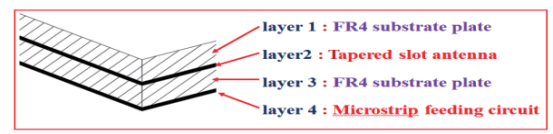

Fig. 8 Side view of the structure of the PCB.

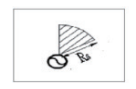

(I)

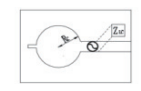

(II)

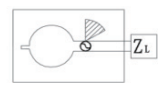

(III)
Fig. 9 Structure of sector coupling and feeding circuits.

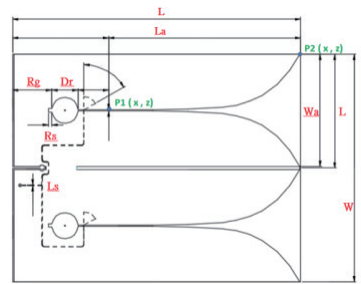

Fig. 10 Antenna structure and pattern design.

\section{Experiment and Improvement}

In this study, the PCB dielectric constant used was $\varepsilon$ r $=4.4$, the FR4 substrate thickness was $1.6 \mathrm{~mm}$, and the microstrip line thickness was $0.1 \mathrm{oz}$. The bottom surface in Fig. 8 is a copper-clad conductor and the black solid line in the center represents the power splitter and signal feeding microstrip line on the other side of the PCB. The signal feeding microstrip line crossed the exponential tapered slot line at $90^{\circ}$ and was welded on the L-shaped SAMJ joint on the PCB. Next, the microstrip lines were connected to coaxial cables to feed signals. Although the structure of the proposed antenna was simple, the power splitter and feeding circuit were difficult to design and adjust. Differences in the design of the feeding circuit may influence operating bandwidth and insertion losses. The antenna design and adjustment were completed using the radiofrequency simulation software SEMCAD developed by Schmid \& Partner Engineering AG (SPEAG). After numerous size changes and computations, a set of values that can satisfy the required structure dimensions were obtained as follows (Table 1.):

Table I. Antenna structure dimensions.

\begin{tabular}{|l|l|}
\hline Single antenna length: & $\mathrm{L}=180 . \mathrm{mm}$ \\
\hline Antenna array width: & $\mathrm{W}=140 . \mathrm{mm}$ \\
\hline Slot width: & $\mathrm{Wa}=70 . \mathrm{mm}$ \\
\hline Tapered line length: & $\mathrm{La}=120 . \mathrm{mm}$ \\
\hline Diameter of matching cavity: & $\mathrm{Dr}=16.5 \mathrm{~mm}$ \\
\hline Length of circular cavity groove: & $\mathrm{Rs}=2 . \mathrm{mm}$ \\
\hline Distance between the cavity and the back end: & $\mathrm{Rg}=23 \mathrm{~mm}$ \\
\hline Angle of the feeding circular sector: & $\mathrm{Pa}=55$ \\
\hline Width of the primary microstrip feeding line: & $\mathrm{LS}=0.75 \mathrm{~mm}$ \\
\hline
\end{tabular}

Substituting these parameters into simulation yields the antenna voltage standing wave ratio (VSWR) curves at all bandwidths and satisfactory values (Fig. 11). The simulation results revealed that the VSWR was equal to or lower than 2.5 with the operating bandwidth of $0.7-6.0 \mathrm{GHz}$. The isolation of the antenna at nearly all bandwidths was lower than $-35 \mathrm{~dB}$ (Fig. 12) and achieved $-40 \mathrm{~dB}$ at $2.1 \mathrm{GHz}$ or higher frequencies. The isolation of horizontal and vertical polarization poles exhibited minimum mutual influence. Some dimensions of the antenna were modified to improve the antenna characteristics. The L, W, Wa, La, R, and Pa dimensions of the antenna affected antenna characteristics. Considering the error effects on antenna characteristics, this study calculated the influence of adding or subtracting 0.1 $\mathrm{mm}$ to or from the signal feeding microstrip size, microstrip feeding extrusion length, and matching cavity diameter on the antenna VSWR. The results showed that within the bandwidths of $0.7-6.0 \mathrm{GHz}$, the return loss variation of the antenna was minimal. Slight differences were observed before and after frequency point movements, as shown in Figs. 9-1, 9-2, and 9-3.

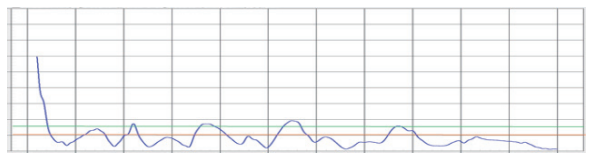

Fig. 11 VSWR of final outcome after parameter optimization at $0.7-6.0 \mathrm{GHz}$.

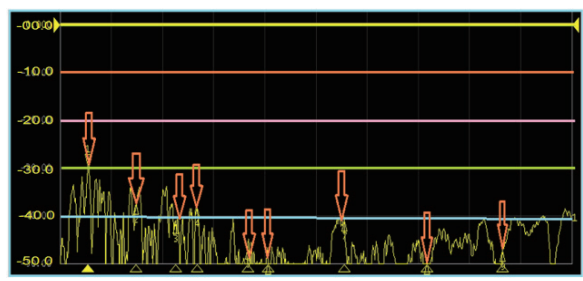

Fig. 12 Isolation of $\mathrm{V} / \mathrm{H}$ ports at $0.7-6.0 \mathrm{GHz}$.

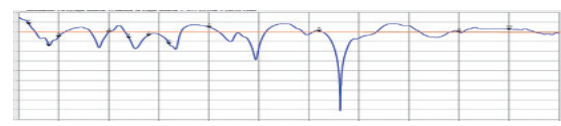

Fig. 13 Return loss without modification.

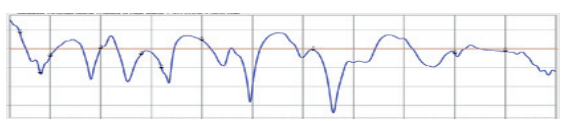

Fig. 14 Return loss after the first modification. 


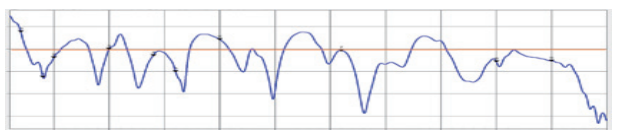

Fig. 15 Return loss after the second modification.

\section{Data Analysis}

This study modified microstrip antennas several times according to the aforementioned dimensions. The parameters and dimensions affected the VSWR of a real antenna. Figs. 9-1-9-3 show that the enlarged circular cavity structures improved the performance at the high-frequency end. Return losses at all frequency points were lower than $-8 \mathrm{~dB}$, satisfying the experimental requirements. In this study, SEMCAD was used to simulate the performance of the proposed antenna at various bands stipulated in currently effective mobile communications regulations. Five frequency points that represent low, medium, and high bands $(0.9 \mathrm{GHz}, 1.7 \mathrm{GHz}, 2.1 \mathrm{GHz}, 3.6 \mathrm{GHz}$, and $5.5 \mathrm{GHz})$ were selected to simulate the performance of the antenna encompassed by EM wave absorbers, as shown in Figs. 1718.

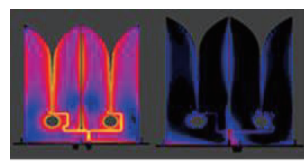

Fig. 16 Current distribution of the cross polarized planes of the antenna at $0.9 \mathrm{GHz}$.
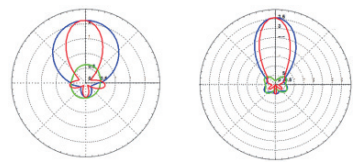

Fig. 17. Radiation patterns at $0.9 / 1.7 \mathrm{GHz}$.
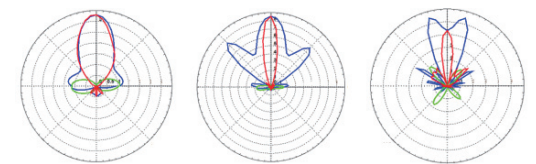

Fig. 18 Radiation patterns at $2.1 / 3.6 / 5.5$ / GHz.

\section{Conclusions}

This study used the SEMCAD EM simulation software developed by SPEAG to optimize the proposed antenna. Based on the Long-Term Evolution Advanced mobile communications standards, five frequency points that represent low, medium, and high bands were selected for the simulation. Relevant antenna performance parameters were completed as follows: The antenna operating bandwidth VSWR (Fig. 9), isolation of dualpolarized antennas, the antenna main body, current distribution of the cross- polarization planes (Fig. 16), and the $3 \mathrm{D}$ and $2 \mathrm{D}$ radiation efficiency patterns of the antenna. The experimental data indicated that the antenna performance within the $0.7-6.0 \mathrm{GHz}$ band satisfied the benchmark requirements of broadband antennas.

The power splitter and coupling feeding circuit structure adopted in this study are simple. However, the distance between the microstrip lines and exponentially tapered cavity grooves is a crucial area that requires further attention. If the distance between the feeding microstrip line and the cavity groove, as well as the diameter of the circular cavity can be further modified, the impedance property of the antenna can be improved; this requires further research. Moreover, the thickness of the PCB substrates and selection of the relative dielectric constants affected the antenna "es dimensions and performance. The proposed antenna did not use PCB substrates with high dielectric constants. If such materials were used to design the antenna, a lower operating bandwidth could have been achieved under an identical volume without compromising the performance requirements.

This study analyzed the EM performance of $2 \times 2$ Vivaldi broadband antenna arrays. The simulation results revealed that the characteristic data obtained were extraordinary. The proposed antenna arrays were planned to be applied in a MIMO OTA EM wave anechoic chamber with multiple antennas. The results revealed that the antenna possessed outstanding power splitter and coupling feeding circuit designs that reduced insertion losses while maintaining excellent radiative directivity. The compactness and small cross-section area along the propagation direction considerably reduced multipath reflections and interferences of the multiantenna systems. This study used pyramidal EM wave absorbers to surround the antenna array adequately to reduce the lateral and backward radiation from the designated radiation direction at $30^{\circ}$ of the half-power beam width or higher. This approach reduced the multipath reflections and interferences of the antennas in nonreflective indoor environments. Introducing the proposed antenna arrays into existing MIMO OTA measurement systems may reduce the multipath reflections produced by lateral radiation, thereby satisfying the MIMO OTA requirements and generating accurate measurements. The proposed antenna arrays should contribute substantially in the mobile communications field in terms of MIMO OTA measurements.

\section{References}

[1] Y. Q. Yang, C. M. Zhang, S. Lin and A. E. Fathy, "Development of An Ultra Wideband Vivaldi Antenna Array," in Proc. Antennas and Propagation Society International Symposium, July. 2005, pp. 606-609.

[2] P. J. Gibson, "The Vivaldi Aerial," in Proc. 9th European Microwave Conf, Brighton, UK, 1979, pp. 101-105.

[3] D. H. Schaubert and T. H. Chio, "Wideband Vivaldi Arrays for Large Aperture Antennas," in Proc. Perspective on Radio Astronomy Technologies for Large Antenna Arrays of Conf, Haryana, India, Apr. 1999, pp. 49-57.

[4] W. K. Chen, "Theory and Design of Broadband Matching Networks," Pergamon Press, 1976.

[5] J. Zhang, E. C. Fear and R. H. Johnston, "Cross-vivaldi Antenna for Breast Tumor Detection," Wiley's Microw. Opt. Technol. Lett., vol. 51, no. 2, pp. 275-280, Feb. 2009.

[6] G. Adamiuk, T. Zwick and W. Wiesbeck, "Compact Dualpolarized UWB Antenna Embedded in A Dielectric," IEEE Trans. Antennas Propag., vol. 58, no. 2, pp. 279-286, Feb. 2010.

[7] J. Shin and D. H. Schaubert, "A Parameter Study of Stripline-fed Vivaldi Notch-antenna Arrays," IEEE Trans. on Antenna and Propagation, vol. 47, no. 5, May. 1999, pp. 879-886.

[8] E. Gazit, „Improved Design of The Vivaldi Antenna,” in Proc Microwaves, Antennas and Propagation, IEEE Proceedings $H$, vol. 135, no. 2, pp. 89-92, Apr. 1988. 